\title{
Relação entre estadiamento, tratamento e sobrevida de pacientes com câncer gástrico
}

\author{
Relationship between staging, treatment and survival of gastric cancer patients \\ Relación entre estadificación, tratamiento y supervivencia de pacientes con cáncer gástrico \\ Clarissa Porfírio Mendes ${ }^{1 *}$, Jamil Michel Miranda do Vale ${ }^{1}$, Marcos José Risuenho Brito Silva², Mary \\ Elizabeth de Santana², Alzinei Simor².
}

\section{RESUMO}

Objetivo: Descrever a relação entre o estadiamento, tratamento e sobrevida de pacientes com câncer gástrico submetidos ao tratamento cirúrgico. Métodos: Estudo retrospectivo, quantitativo, realizado em um centro de alta complexidade oncológico da região norte do Brasil. A análise ocorreu por meio da estatística descritiva com método de Kaplan-Meier, Qui-quadrado e o Log-rank. Analisados 135 prontuários no período de janeiro de 2008 a janeiro de 2015. Resultados: Apresentaram com maior frequência no Estadiamento IV (37,0\%); a Gastrectomia Total $(51,1 \%)$ foi a mais realizada entre os pacientes estudados seguida pela Gastrectomia Subtotal $(23,7 \%)$, em relação ao tratamento adjuvante a maioria dos pacientes não realizou (74,8\%); Quanto à sobrevida geral do paciente submetido à cirurgia por câncer gástrico foi de 36,8\% em cinco anos. Em relação à sobrevida e o tratamento indicou não haver evidências estatísticas significativas, em contrapartida, a sobrevida e estadiamento houve fortes evidências estatísticas. Conclusão: O câncer gástrico está sendo detectado tardiamente, acarretando uma série de problemas ao paciente, a sua família e à sociedade. Por isso, importante refletir sobre a caracterização do tratamento e estadiamento e a relação dessas variáveis com a sobrevida desse paciente, a fim de ampliar as evidências científicas e fornecer subsídios que direcionem políticas públicas.

Palavras-chave: Neoplasias gástricas, Análise de sobrevida, Estadiamento de neoplasias, Terapia combinada.

\begin{abstract}
Objective: Describe the relationship between staging, treatment and survival of patients with gastric cancer undergoing surgical treatment. Methods: Retrospective, quantitative study, carried out in a highly complex oncology center in the northern region of Brazil. The analysis took place using descriptive statistics using the Kaplan-Meier method, Chi-square and Log-rank. 135 medical records were analyzed in the period from January 2008 to January 2015. Results: They presented more frequently in Stage IV $(37,0 \%)$; Total Gastrectomy $(51,1 \%)$ was the most performed among the studied patients followed by Subtotal Gastrectomy $(23,7 \%)$, in relation to adjuvant treatment, most patients did not perform it $(74,8 \%)$; As for the overall survival of the patient who underwent surgery for gastric cancer, it was $36,8 \%$ in five years. Regarding survival and treatment, there was no statistically significant evidence. On the other hand, survival and staging had strong statistical evidence. Conclusion: Gastric cancer is being detected late, causing a series of problems for the patient, his family and society. Therefore, it is important to reflect on the characterization of treatment and staging and the relationship of these variables with the survival of this patient, in order to expand the scientific evidence and provide subsidies that guide public policies.
\end{abstract}

Key words: Stomach neoplasms, Survival analysis, Neoplasm staging, Combined modality therapy.

\footnotetext{
${ }^{1}$ Universidade Federal do Pará (UFPA), Belém - PA. *E-mail: clarissapmendes@yahoo.com.br

2 Universidade do Estado do Pará (UEPA), Belém - PA.
} 


\section{RESUMEN}

Objetivo: Describir la relación entre estadificación, tratamiento y supervivencia de pacientes con cáncer gástrico sometidos a tratamiento quirúrgico. Métodos: Estudio retrospectivo, cuantitativo, realizado en un centro oncológico de alta complejidad en la región norte de Brasil. El análisis se realizó mediante estadística descriptiva mediante el método de Kaplan-Meier, Chi-cuadrado y Log-rank. 135 registros analizados en el período de enero de 2008 a enero de 2015. Resultados: Se presentaron con mayor frecuencia en el estadio IV (37,0\%); La Gastrectomía Total $(51,1 \%)$ fue la más realizada entre los pacientes estudiados seguida de la Gastrectomía Subtotal $(23,7 \%)$, en relación al tratamiento adyuvante, la mayoría de los pacientes no lo realizaron (101; 74,8\%); En cuanto a la supervivencia global del paciente intervenido de cáncer gástrico, fue del $36,8 \%$ en cinco años. En cuanto a la supervivencia y el tratamiento, no hubo evidencia estadísticamente significativa, mientras que la supervivencia y la estadificación tuvieron una fuerte evidencia estadística. Conclusión: El cáncer de estómago se está detectando de forma tardía, provocando una serie de problemas para el paciente, su familia y la sociedad. Por ello, es importante reflexionar sobre la caracterización del tratamiento y la estadificación y la relación de estas variables con la supervivencia de este paciente, con el fin de ampliar la evidencia científica y brindar subsidios que orienten las políticas públicas.

Palabras clave: Neoplasias gástricas, Análisis de supervivencia, Estadificación de neoplasias, Terapia combinada.

\section{INTRODUÇÃO}

No campo da pesquisa, a epidemiologia do câncer tem alcançado sucesso e prestígio ao demonstrar o papel de vários fatores de risco e de proteção na cadeia causal de diversos tipos de neoplasias malignas, bem como tem fornecido evidências científicas na formulação de estratégias para a prevenção e controle do câncer (FABIANI L, et al., 2019).

A sobrevida de pacientes a partir de dados provenientes de registros de câncer, de base populacional ou hospitalar, ganha um papel de destaque ao direcionar a formulação de Políticas Públicas de Saúde. Haja vista que, partindo desse dado, é possível visualizar fatores que permitirão ampliar a qualidade de vida do paciente após o tratamento (BERGERON-BOUCHER MP, et al., 2019).

Neste ínterim, o Câncer Gástrico (GC) representa a terceira neoplasia maligna mais comum no cenário nacional e uma importante causa de morte em todo o mundo. Estima-se que a probabilidade de sobrevida seja em torno de $30 \%$. Esse dado está relacionado com aumento da expectativa de vida acrescido das modalidades de tratamento oncológico; com a realização da detecção precoce e rastreamento; diagnóstico precoce e intervenção menos radical (HOWLADER N, et al., 2017; JEMAL A, et al., 2017; STEWART BW e WILD CP, 2015; RAMOS MFKP, et al., 2018).

O CG possui diversos fatores de risco, dentre eles estão fatores modificáveis e não modificáveis. Um fator que ganha destaque é a infecção por Helicobacter pylori, a qual é considerada a causa mais fortemente associada ao desenvolvimento de CG. As populações de menor condição social podem apresentar maior prevalência de infecção por Helicobacter pylori, assim como tabagismo e dieta com deficiências nutricionais constituem-se fatores comumente relacionados ao desenvolvimento do CG (ALICANDRO G, et al., 2017; DIACONU S, et al., 2017).

O tumor geralmente é diagnosticado em avançado estágio dada à agressividade do processo carcinogênico, em que os adoecidos têm uma sobrevivência média de $20 \%$ em cinco anos. Contudo, é importante ressaltar que caso diagnosticado precocemente possui grande potencial para cura, apresentando uma média de sobrevida em cinco anos superior a 90\% (ALMEIDA MFA, et al., 2018).

A partir do diagnóstico, a conduta é definida de acordo com o estadiamento da doença, dada pela Classificação de Tumores Malignos (TNM), considerando o avanço do tumor primário, linfonodos acometidos e metástases. No caso do câncer gástrico, a classificação estágio 0 é indicada para apresentações iniciais e 
nos casos mais avançados variam de estágio 1 a 4 . Essa classificação indicará as diferentes modalidades terapêuticas, como ressecção endoscópica, ressecção cirúrgica, radioterapia e quimioterapia. No caso, do tumor ressecável, a gastrectomia ainda é a referência ainda amplamente aceita no meio acadêmico (JURADO-MUÑOZ PA, et al, 2021; HOYOS-VALDELAMAR JC, et al, 2020).

A mudança no perfil de conduta clínica e morbimortalidade no mundo e o perfil demográfico do Brasil, ocasionou o aumento significativo do número de novos casos de câncer (INCA, 2019). A partir da valorização do rastreamento precoce do câncer gástrico, motivando à realização de novos estudos elaboramos o seguinte questionamento: Qual a probabilidade acumulada de sobrevida do paciente submetido à cirurgia por câncer gástrico em um hospital referência em oncologia da região norte?

Posto isto, este estudo tem como objetivo analisar a relação entre o estadiamento, tratamento e sobrevida do paciente submetido à cirurgia por câncer gástrico em um hospital referência em oncologia da região norte.

\section{MÉTODOS}

Trata-se de um estudo descritivo retrospectivo e inferencial, dos dados clínico-epidemiológicos acerca da sobrevida de 135 pacientes submetidos à cirurgia por câncer gástrico, entre janeiro de 2008 e janeiro de 2015.

O estudo foi realizado em um hospital de referência em oncologia, situado em Belém, no Pará. A coleta de dados ocorreu na Divisão do Arquivo Médico e Estatístico (DAME), tendo como fontes de informações os prontuários.

Nos critérios de inclusão, os prontuários de pacientes deveriam conter diagnóstico histológico comprovado de câncer gástrico e que não tivessem realizado tratamento cirúrgico por câncer gástrico anteriormente. Não compuseram a amostra os prontuários de pacientes que evoluíram a óbito sem terem sido submetidos a nenhum tratamento.

O projeto foi aprovado pelos Comitês de Ética e Pesquisa do Hospital Ophir Loyola e do Instituto Ciências da Saúde da Universidade Federal do Pará, sob o número 1.209.290. Para coleta de dados utilizamos formulário com as seguintes variáveis clínicas: anatomopatológico, estadiamento (TNM), tipo de cirurgia, complicações, tratamento adjuvante, fatores de risco e sintomas clínicos antes da confirmação do diagnóstico.

Para análise dos dados ocorreu por intermédio da estatística descritiva e inferencial, sendo aplicados os testes estatísticos não-paramétricos, como o Qui-quadrado de aderência para proporções esperadas iguais e Log-rank, todos aplicados ao nível de significância de $p<0,05$, ou seja, para avaliar a significância estatística procedemos tal como na análise clássica, analisando o valor de $p$.

A análise univariada da sobrevida deu-se pelo método de Kaplan-Meier, por intermédio do cálculo do tempo de seguimento e da sobrevida global (expressa em anos), foi utilizada o intervalo e entre o tratamento cirúrgico e o último atendimento hospitalar, com o objetivo de avaliar provável óbito ou abandono, considerando um tempo de sobrevida de 5 anos. Foi realizada a estatística descritiva sendo expressa através de média e mediana e seus respectivos desvios-padrão.

Portanto, foram utilizados os softwares Statistical Package for the Social Sciences (SPSS) versão $20.0 \mathrm{e}$ Microsoft Excel para a confecção e análise das curvas de sobrevivência, tendo como base o método de Kaplan-Meier e o teste comparativo de Log-rank.

\section{RESULTADOS}

Foram analisados 135 prontuários de pacientes com câncer gástrico. Destaca-se abaixo a Tabela 1, congregando dados sobre o anatomopatológico, tipo de cirurgia, localização do tumor, estadiamento e realização de quimioterapia adjuvante. 
Tabela 1 - Distribuição do perfil de pacientes quanto ao resultado do anatomopatológico, tipo de cirurgia, localização do tumor, estadiamento e realização de quimioterapia adjuvante.

\begin{tabular}{|c|c|c|}
\hline Variáveis & Quantidade & Percentual \\
\hline \multicolumn{3}{|l|}{ Anatomopatológico } \\
\hline Adenocarcinoma gástrico (AG) & $132^{*}$ & 97,9 \\
\hline Linfoma gástrico (LG) & 2 & 1,4 \\
\hline Tumor estromal gástrico (TEG) & 1 & 0,7 \\
\hline \multicolumn{3}{|l|}{ Tipo de Cirurgia } \\
\hline Gastrectomia Total & $69^{*}$ & 51,1 \\
\hline Gastrectomia Subtotal & 32 & 23,7 \\
\hline Gastroentroanastomose & 08 & 5,9 \\
\hline Laparotomia Exploradora & 26 & 19,3 \\
\hline \multicolumn{3}{|l|}{ Localização do Tumor } \\
\hline Antro & $58^{*}$ & 43,0 \\
\hline Corpo & 35 & 26,0 \\
\hline Fundo & 10 & 7,4 \\
\hline Antro e Corpo & 9 & 6,7 \\
\hline Cárdia & 12 & 8,9 \\
\hline Piloro & 7 & 5,2 \\
\hline Linite Plástica & 4 & 3,0 \\
\hline \multicolumn{3}{|l|}{ Estadiamento } \\
\hline IA & 10 & 7,4 \\
\hline IB & 3 & 2,2 \\
\hline IIA & 12 & 8,9 \\
\hline IIB & 22 & 16,3 \\
\hline IIIA & 21 & 15,6 \\
\hline IIIB & 11 & 8,2 \\
\hline IIIC & 6 & 4,4 \\
\hline IV & $50^{*}$ & 37,0 \\
\hline \multicolumn{3}{|l|}{ Tratamento Adjuvante } \\
\hline Não & $101^{*}$ & 74,8 \\
\hline QT & 34 & 25,2 \\
\hline Total & 135 & 100,0 \\
\hline
\end{tabular}

Legenda: ${ }^{*} p<0,05$ (Teste Qui-quadrado; $\left.p<0,0001\right)$.

Fonte: Mendes CP, et al., 2021.

Quanto à sobrevida dos pacientes, a sobrevida geral do paciente submetido à cirurgia por câncer gástrico foi de $36,8 \%$ em cinco anos; a média de sobrevida foi de 2,4 anos, com erro padrão de 0,2 e intervalo de $95 \%$ de confiança entre 2,0 a 2,9 anos e a mediana foi de 1,7 anos, com erro padrão de 0,5 e intervalo de $95 \%$ de confiança entre 0,8 a 2,7 anos. Então, após cinco anos do procedimento cirúrgico, a probabilidade de sobreviver foi equivalente a $36,8 \%$. O paciente submetido à cirurgia por câncer gástrico sobreviveu em média 2 anos e 4 meses, sendo que a metade dos pacientes sobreviveram até 1 ano e 7 meses. Porém, de acordo com o Gráfico 1 da função de sobrevida dos pacientes submetidos à cirurgia, observa-se que a probabilidade acumulada de estar vivo diminui significativamente até o segundo ano, apresentando tendência de uniformidade a partir do segundo ano em cerca de 40,0\% (Gráfico 1). 
Gráfico 1 - Sobrevida geral do paciente submetido à cirurgia por câncer gástrico

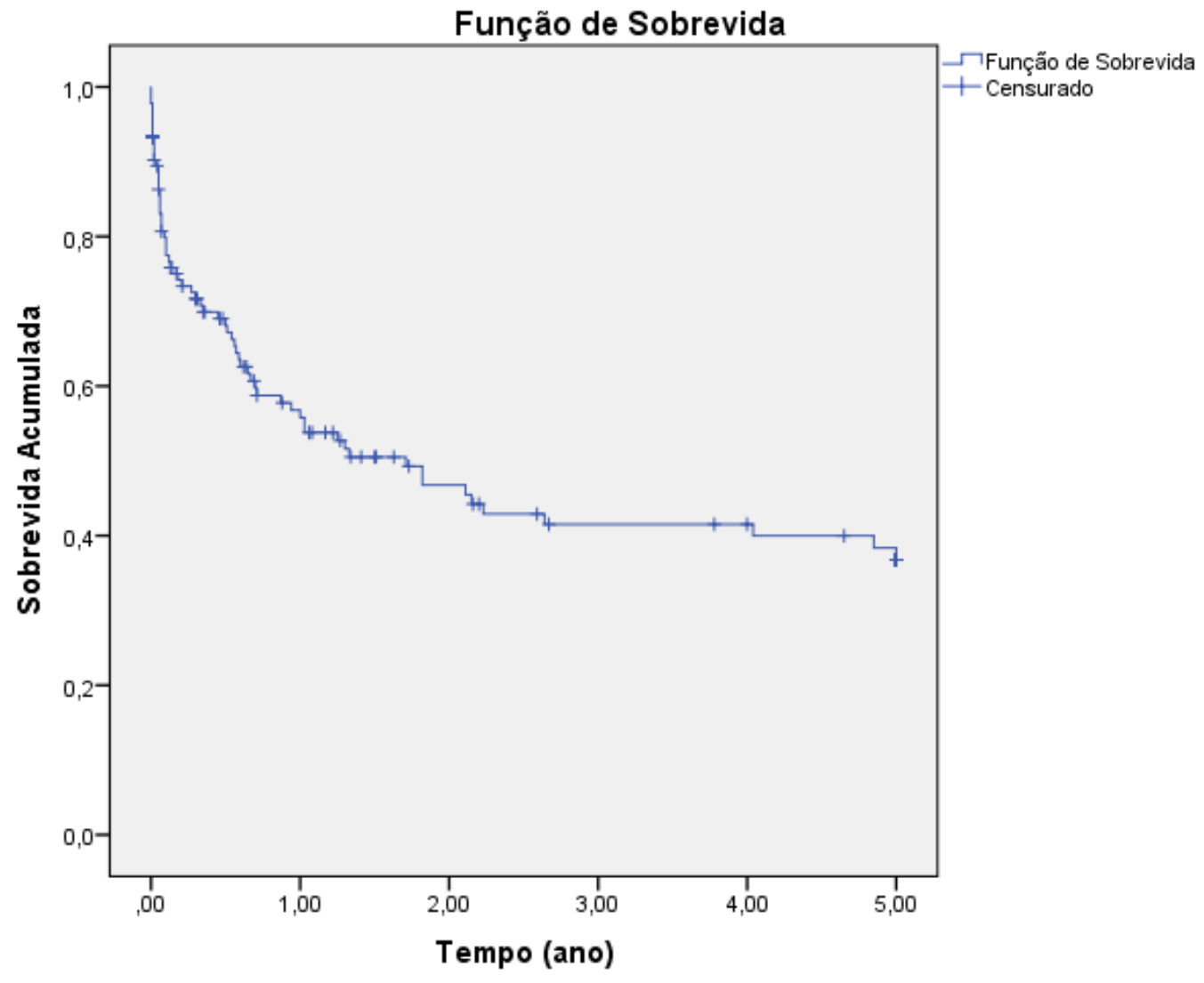

Fonte: Mendes CP, et al., 2021.

Já em relação à sobrevida a partir do tratamento adjuvante, o valor do nível descritivo $(p=0,116)$ do Teste Log-rank indica não haver evidências estatísticas para se sugerir que as diferenças entre as Taxas de Sobrevida dos pacientes estudados são estatisticamente significantes, segundo o fato de ter ou não realizado tratamento adjuvante, ao nível de significância de $p<0,05$ (Tabela 2).

Tabela 2 - Resumo dos valores descritivos para média, mediana e para o teste de Log-Rank

\begin{tabular}{|c|c|c|c|c|c|c|c|c|c|c|}
\hline \multirow{3}{*}{ Variável } & \multicolumn{4}{|c|}{ Média } & \multicolumn{4}{|c|}{ Mediana } & \multirow{3}{*}{$\begin{array}{l}\text { Log- } \\
\text { Rank }\end{array}$} & \multirow{3}{*}{$\begin{array}{c}\text { p- } \\
\text { valo } \\
r\end{array}$} \\
\hline & \multirow[b]{2}{*}{ Valor } & \multirow[b]{2}{*}{$\begin{array}{l}\text { Erro } \\
\text { Padrão }\end{array}$} & \multicolumn{2}{|c|}{$\begin{array}{c}95 \% \text { Intervalo de } \\
\text { confiança }\end{array}$} & \multirow[b]{2}{*}{ Valor } & \multirow[b]{2}{*}{$\begin{array}{l}\text { Erro } \\
\text { Padrão }\end{array}$} & \multicolumn{2}{|c|}{$\begin{array}{l}\text { 95\% Intervalo } \\
\text { de confiança }\end{array}$} & & \\
\hline & & & $\begin{array}{l}\text { Limite } \\
\text { Infe- } \\
\text { rior }\end{array}$ & $\begin{array}{l}\text { Limite } \\
\text { Supe- } \\
\text { rior }\end{array}$ & & & $\begin{array}{c}\text { Limite } \\
\text { Infe- } \\
\text { rior }\end{array}$ & $\begin{array}{l}\text { Limite } \\
\text { Supe- } \\
\text { Rior }\end{array}$ & & \\
\hline \multicolumn{11}{|l|}{ Tipo de Cirurgia } \\
\hline $\begin{array}{l}\text { Gastrectomia } \\
\text { total }\end{array}$ & 2,85 & 0,29 & 2,27 & 3,42 & 2,64 & - & - & - & \multirow{4}{*}{24,762} & \multirow{4}{*}{$\begin{array}{l}p< \\
0,00 \\
01\end{array}$} \\
\hline $\begin{array}{l}\text { Gastrectomia } \\
\text { Subtotal }\end{array}$ & 2,84 & 0,41 & 2,05 & 3,64 & 4,04 & - & - & - & & \\
\hline $\begin{array}{l}\text { Gastroentroana } \\
\text { sto-mose }\end{array}$ & 0,95 & 0,23 & 0,51 & 1,40 & 1,25 & 0,50 & 0,27 & 2,23 & & \\
\hline $\begin{array}{l}\text { Laparotomia } \\
\text { Exploradora }\end{array}$ & 0,63 & 0,33 & 0,00 & 1,28 & 0,21 & 0,16 & 0,00 & 0,52 & & \\
\hline \multicolumn{11}{|c|}{ Tratamento Adjuvante } \\
\hline Não & 2,28 & 0,26 & 1,78 & 2,79 & 0,94 & 0,36 & 0,24 & 1,64 & \multirow{2}{*}{2,477} & 0,11 \\
\hline QT & 2,87 & 0,36 & 2,15 & 3,58 & 2,11 & 0,50 & 1,13 & 3,09 & & \\
\hline
\end{tabular}

Fonte: Mendes CP, et al., 2021. 
Em relação aos pacientes que realizaram quimioterapia tiveram uma média de sobrevida de 2 anos e 2 meses; 6 meses superior à dos pacientes que não realizaram quimioterapia. Assim como, a mediana da sobrevida dos pacientes que realizaram quimioterapia foi de 1 ano e 4 meses a mais do que os pacientes que não realizaram quimioterapia. Logo, quando os pacientes foram submetidos à quimioterapia adjuvante, metade sobreviveram 1 ano e 4 meses a mais do que os pacientes que não realizaram e em média sobreviveram 6 meses a mais (Gráfico 2).

Gráfico 2 - Sobrevida do paciente submetido à quimioterapia adjuvante a partir da cirurgia para câncer gástrico.

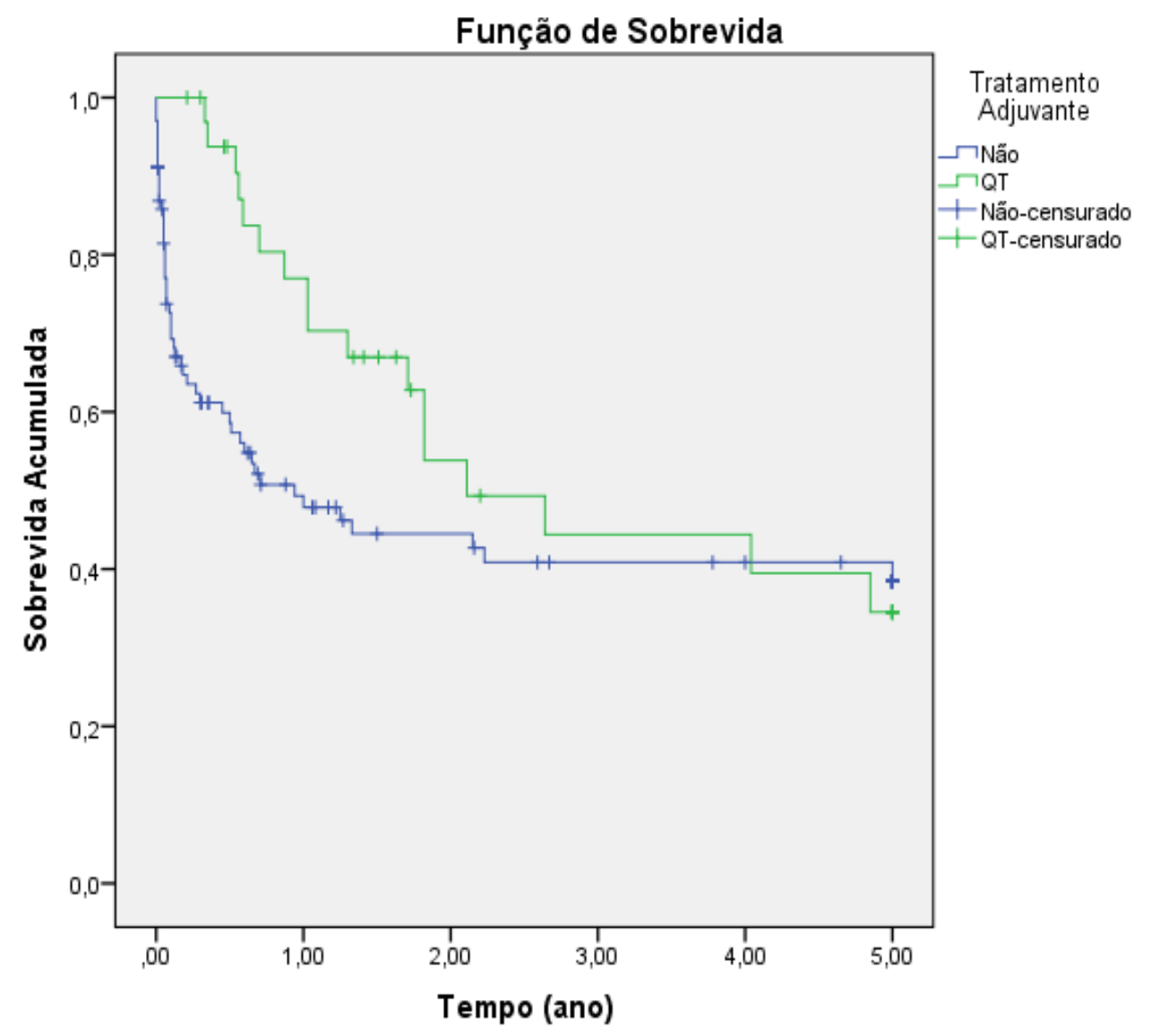

Fonte: Mendes CP, et al., 2021.

Quanto à sobrevida a partir do estadiamento, segundo o nível descritivo $(\mathrm{p}<0,0001)$ do Teste Log-rank há fortes evidências estatísticas para sugerir que a diferença entre as Taxas de Sobrevida é estatisticamente significante segundo o estadiamento, ao nível de significância de 0,05.

Aponta-se que após cinco anos da data do procedimento cirúrgico, dos pacientes com estádio IIIA, dez evoluíram à óbito, dez abandonaram e um sobreviveu; dos pacientes com estádio IIIB, seis evoluíram à óbito, três abandonaram e dois sobreviveram; os com estádio IIIC, quatro evoluíram à óbito, um abandonou e um sobreviveu; e com estádio IV, 36 evoluíram à óbito e 14 abandonaram o tratamento. Ou seja, dos 135 pacientes do estudo, 88 estavam com estadiamento avançado, dentre os quais 56 evoluíram a óbito, 28 abandonaram o tratamento (provavelmente evoluíram à óbito logo em seguida) e 4 sobrevivem.

Já sobrevida dos pacientes com estádio mais precoce (estádio IA, IB e IIA) girou em torno de $90 \%$ em cinco anos; nos estádios IIB, IIIA, IIIB e IIIC girou em torno de 25 a $55 \%$; no entanto, com um quadro bastante diferente no estádio IV, pois os pacientes não sobreviveram até os cinco anos abordados no estudo e cuja grande maioria ( $80 \%$ dos pacientes) evolui a óbito no primeiro ano após à cirurgia por câncer gástrico e $20 \%$ até o segundo ano após a cirurgia, retratando um prognóstico extremamente reservado (Gráfico 3). 
Gráfico 3 - Sobrevida do paciente submetido à cirurgia por câncer gástrico a partir do estadiamento.

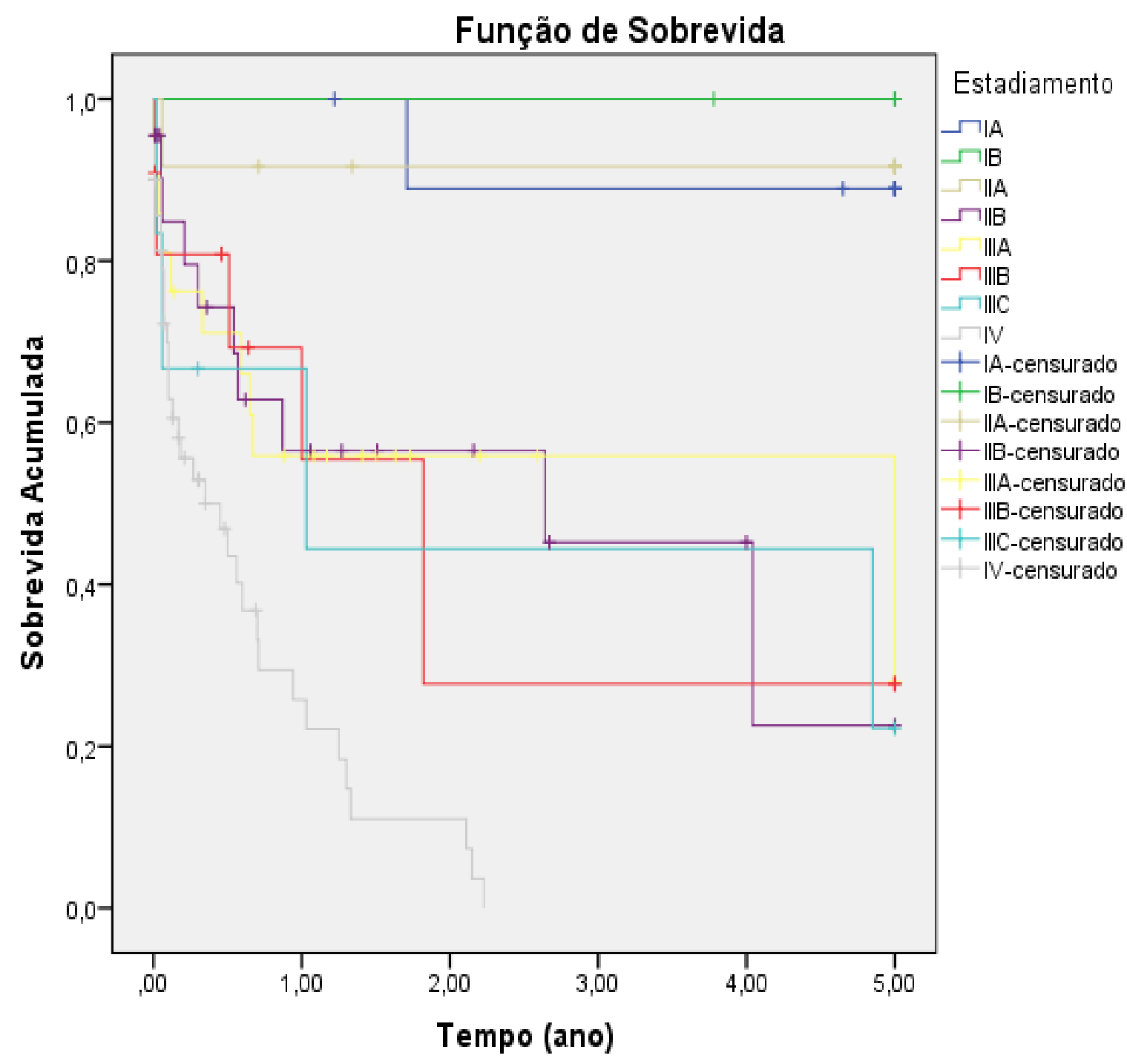

Fonte: Mendes CP, et al., 2021.

Logo, a maioria dos pacientes que foi submetida a um procedimento cirúrgico por câncer gástrico encontrava-se em estadiamento avançado $(65,2 \%)$, notando-se uma relevante diferença da sobrevida em pacientes com câncer gástrico precoce e os que têm câncer gástrico avançado, pois quanto mais precoce foi o estadiamento do paciente com câncer gástrico, melhor foi sua sobrevida.

Por último, a sobrevida a partir do tipo de cirurgia, obteve o nível descritivo $(p<0,0001)$ do Teste Log-rank com fortes evidências estatísticas para se sugerir que a diferença entre as Taxas de Sobrevida seja estatisticamente significante, segundo o Tipo de Cirurgia a que foi submetido o paciente, no contexto amazônico, ao nível de significância de 0,05.

Denota-se que os pacientes que realizaram laparotomia exploradora sobreviveram até 8 meses após o procedimento cirúrgico e os que realizaram gastroenteroanastomose sobreviveram até um ano e quatro meses após a cirurgia. Bem como, nota-se que os pacientes submetidos à gastrectomia total têm um traçado na função de sobrevida semelhante aos dos pacientes submetidos à gastrectomia subtotal, com sobrevida global em cinco anos de 43\% (Gráfico 4). 
Gráfico 4 - Sobrevida do paciente submetido à cirurgia por câncer gástrico a partir do tipo de cirurgia.

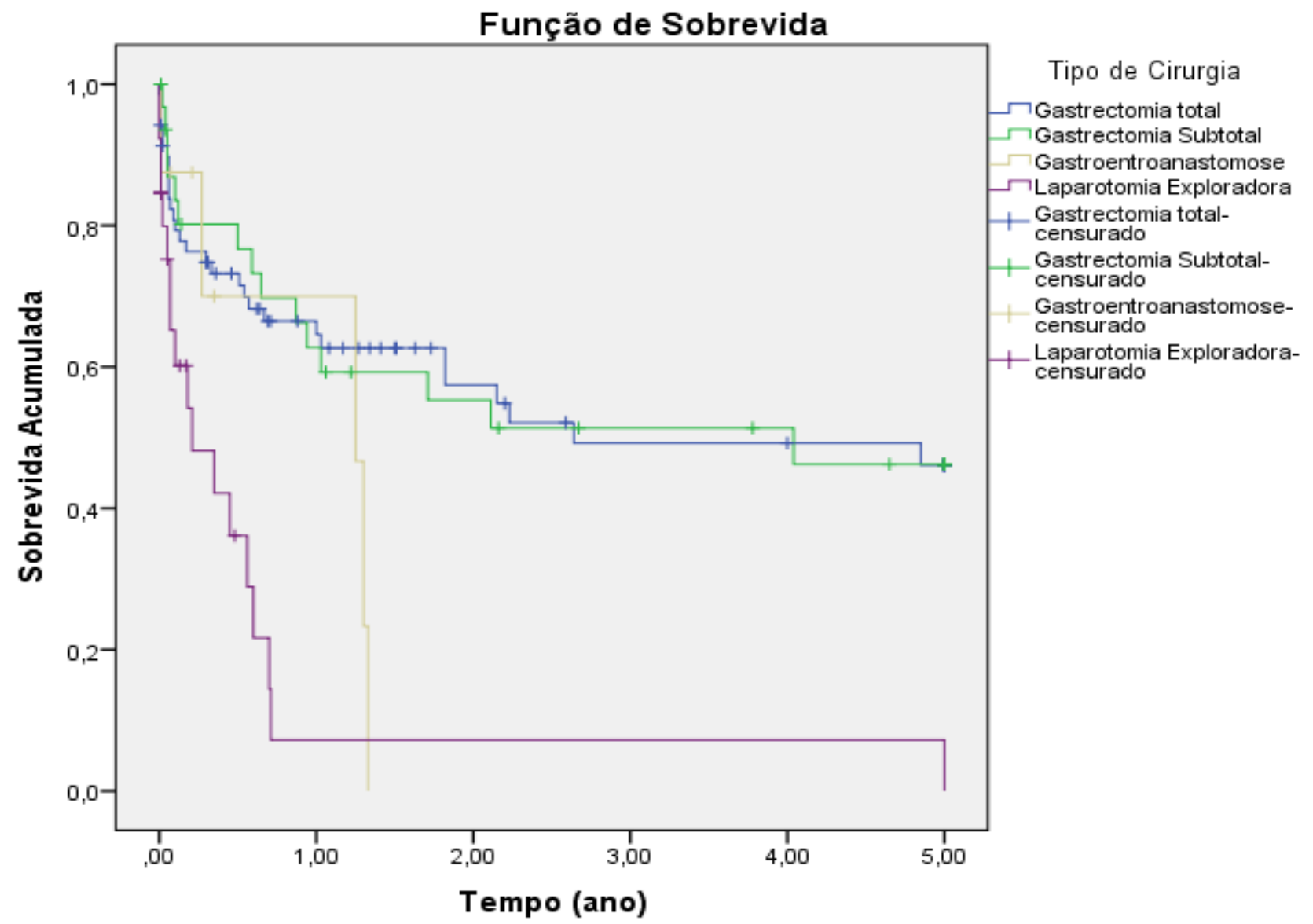

Fonte: Mendes CP, et al., 2021.

\section{DISCUSSÃO}

O tratamento cirúrgico no câncer gástrico vem ganhando reconhecimento como mecanismo de prognóstico favorável, com taxas de sobrevivência que vem cada vez mais aumentando, justamente em virtude da inserção de novas técnicas e melhorias dentro do processo operatório. Tal fato, tem como resultado a prolongação da sobrevida do paciente, mesmo em casos que eram considerados incuráveis (RAMOS MFKP, et al., 2019).

As possibilidades terapêuticas e a sobrevida dos pacientes acometidos pelo câncer gástrico estão diretamente relacionadas ao estadiamento no momento do diagnóstico e o início oportuno do tratamento. No entanto, ainda se depara com o início de tratamento tardio, sendo assim, torna-se fundamental a efetivação de políticas públicas nesse sentido e o empenho dos profissionais de saúde, oncologistas e não oncologistas, na intervenção precoce desses tumores (FIGUEROA-GIRALT M, 2018).

Para a realização do tratamento, uma das etapas essenciais é o estadiamento, o qual pode ser realizado antes da cirurgia ou em casos mais graves no próprio ato cirúrgico. No que concerne ao estadiamento do presente estudo, identificou uma taxa significativa de pacientes em estagio IV (37\%), fato que congrega com o estudo de Chen $\mathrm{K}$, et al. (2015) que evidencia uma grande parcela de pacientes operados com estadiamento avançado, pois entre as 294 amostras, 6 (2,1\%) foram estádio I, 85 (28,9\%) eram estádio II, 97 (33,0\%) eram estádio III, e 106 (36,1\%) estavam em estágio IV.

De acordo com os achados histopatológicos, o câncer gástrico se apresenta de três formas distintas: adenocarcinoma (responsável por 95\% dos tumores), linfoma (diagnosticado em cerca de $3 \%$ dos casos) e leiomiossarcoma, iniciado em tecidos que dão origem aos músculos e aos ossos (INCA, 2019). 
O tipo histológico mais comum, em mais de $90 \%$ dos casos, é o Adenocarcinoma. O que corrobora com o presente estudo, em que a forma predominantemente encontrada foi o Adenocarcinoma. O adenocarcinoma gástrico tem incidência duas vezes maior em homens, apresentando com maior frequência o estágio III, seguido do II (INCA, 2019; JURADO-MUÑOZ PA, et al., 2021; HOYOS-VALDELAMAR JC, et al., 2020).

$\mathrm{Na}$ literatura analisada, percebe-se uma maior abordagem sobre as gastrectomias, porém é relevante apontar que neste estudo houve um índice elevado de pacientes que foram submetidos somente à gastroenteroanastomose (5,9\%), por terem uma doença irressecável ou até mesmo à laparotomia exploradora (19,3\%), por não ter muito a fazer devido à extensão do tumor ou às metástases para órgãos adjacentes. Porém, a maioria dos estudos publicados sobre a abordagem cirúrgica no câncer gástrico é de gastrectomias laparoscópicas subtotais (JURADO-MUÑOZ PA, et al., 2021; SZOR DJ, et al., 2020)

Referente ao tratamento adjuvante realizado observa-se que também correspondem aos outros encontrados no meio científico. Em estudo realizado com 490 pacientes com CG apenas 10,42\% apresentaram terapia dupla, cirurgia e quimioterapia. A não significância estatística da quimioterapia adjuvante na sobrevida, no estudo, pode ter ocorrido pelo fato da maioria dos pacientes que não terem sido submetidos à quimioterapia adjuvante $(74,8 \%)$, quadro bastante distante da realidade japonesa, em que dos 123 pacientes submetidos à cirurgia por câncer gástrico, somente 13 pacientes não receberam quimioterapia $(10,5 \%)$ pós-operatória, devido ao agravamento do estado de desempenho do paciente (MONTALVÁN EE, et al., 2017).

Estudo demonstra que $45(51,7 \%)$ dos pacientes submetidos à terapia adjuvante com quimiorradioterapia mostraram sobrevida maior pela curva de Kaplan-Meier quando comparados a outros $33(48,3 \%)$ com terapia incompleta ou que se recusaram a iniciar a terapia adjuvante. Percebeu-se também que o prognóstico foi influenciado pelo acometimento linfonodal, em que a média de linfonodos ressecados foi de $30,8 \%$, impactando na sobrevida dos pacientes (ANDREOLLO NA, et al., 2019).

Assim, podemos pensar na diminuição dos impactos das complicações do pós-operatório ao prognóstico, em longo prazo, a quimioterapia neoadjuvante e quimioterapia adjuvante para pacientes que desenvolverem complicações pós-operatórias (WANG S, et al., 2019).

Outro ponto importante a ser levantado dentro do tratamento se refere ao seguimento do paciente, visto que a taxa de recidiva é alta, sendo maior nos primeiros três anos, necessitando de monitoramento sistemático por meio de anamnese e exame físico a cada 4 meses no primeiro ano. A endoscopia digestiva alta está indicada para pacientes submetidos à gastrectomia parcial, já outro exame obedece a indicação do consenso clínico, não existindo uma regularidade uma vez que a taxa de sobrevida em 5 anos do diagnóstico é menor de 30\% (SMYTH EC, et al., 2016).

Todavia, os sintomas do câncer gástrico não são específicos, o que dificulta o diagnóstico precoce. Sintomas como massa palpável no abdome superior, hepatomegalia, linfonodomegalia supraclavicular e periumbilical, mais específicos, já sugerem doença avançada, com presença de metástase à distância (INCA, 2019).

A sobrevida geral do paciente submetido à cirurgia por câncer gástrico na pesquisa foi de $36,8 \%$ em cinco anos. Entretanto, estudos acerca dos pacientes com câncer gástrico apontam taxas de sobrevida global, após cinco anos, superiores a $80 \%(p=0,31)$; taxa bastante distanciada da apontada no contexto amazônico, de $36,8 \%$ (NORERO E, et al., 2019).

Corroborando com tal informação, a experiência de outros países da América Latina, como o Paraguai, tem demonstrado índice de sobrevida global igual a $88 \%$, o que, associado a um tratamento oncológico cirúrgico adequado e padronizado em todos os centros de tratamento referenciados, leva a índices de sobrevida global em cinco anos superior a $90 \%$ e, por consequência, a diminuição significativa dos custos de saúde (MONTIEL-ROA AJ, et al., 2019).

Após o tratamento, que pode ser cirúrgico ou endoscópico, contudo, o prognóstico varia de acordo com diversos fatores: idade, sexo, tipos histológicos, nível socioeconômico. Contudo, o maior deles é o 
estadiamento no momento do diagnóstico que, infelizmente, junto com o tratamento não obedecem à padronização no Brasil (TONETO MG e VIOLA L, 2018).

O estudo realizado por Chen K, et al. (2015) demonstrou que os dados de acompanhamento clínico estavam completos com 284 participantes, em uma série de 25,1 meses ( 2 anos e 1 mês), houve 32 óbitos $(11,3 \%)$ e 252 sobreviventes (88,7\%). Quadro diferente do apresentado nesta pesquisa, que após dois anos, apontava um cenário com cerca de $60 \%$ dos pacientes já tendo evoluído a óbito e com cerca de $40 \%$ de sobreviventes.

No ocidente, a sobrevida em cinco anos é em torno de 30\% nos países desenvolvidos e $20 \%$ nos países em desenvolvimento, mostrando que os achados desta pesquisa estão na faixa de sobrevida indicada na literatura. Uma possível explicação para as diferenças nas taxas de mortalidade entre os países asiáticos e o resto do mundo, está na intervenção em estágios iniciais e em um maior volume de pessoas, o que se traduz em uma experiência cirúrgica superior (JURADO-MUÑOZ PA, et al., 2021).

Costa WL, et al. (2015) demonstraram em um estudo realizado em São Paulo que a sobrevida média foi de 56 meses (4 anos e 6 meses) e em 5 anos a sobrevida global foi de 49,2\%. Logo, mais que o dobro da sobrevida média apresentada, que foi de 2 anos e 4 meses e com 12,4\% a mais de pacientes sobrevivendo após cinco anos da cirurgia.

Um aspecto importante que deve ser destacado se refere à curva de sobrevida dos pacientes com mais de 5 anos, que tende a achatar entre o $5^{\circ}$ e $10^{\circ}$ ano de seguimento pós-operatório, denotando que o principal período de mortalidade desta população esta nos dois primeiros anos de pós-operatório (FIGUEROAGIRALTM, 2018).

Portanto, destaca-se que países que investem menos na detecção precoce do câncer gástrico, inevitavelmente, aumentam seus custos com a saúde ao ter que tratar a doença responsável pela segunda maior taxa de mortalidade por câncer no Brasil e no mundo, ficando atrás somente do câncer de pulmão.

\section{CONCLUSÃO}

A partir da realização do presente estudo, foi possível analisar a relação entre o estadiamento, tratamento e sobrevida do paciente submetido à cirurgia por câncer gástrico. Identificou-se que os pacientes com câncer gástrico são admitidos em estadiamento avançado, sendo submetidos às cirurgias paliativas, o que impacta diretamente na sobrevida desses pacientes, tendo somente $36,8 \%$ dos pacientes sobrevivendo após 5 anos da realização do procedimento cirúrgico. Diante do cenário apresentado, faz-se necessário favorecer um maior alcance das políticas de prevenção e tratamento precoce, bem como mudanças de estratégias institucionais, a fim de corroborar à redução das taxas de câncer gástrico, direcionar estratégias que favoreçam o tratamento precoce e assistência integral, aumentando significativamente a sobrevida paciente submetido à cirurgia por câncer gástrico, podendo trazer benefícios à sociedade como um todo.

\section{REFERÊNCIAS}

1. ALICANDRO G, et al. Educational inequality in cancer mortality: a record linkage study of over 35 million Italians. Cancer Causes Control, 2017; 28 (9): 997-1006.

2. ALMEIDA MFA, et al. Computed tomography with a stomach protocol and virtual gastroscopy in the staging of gastric cancer: an initial experience. Revista radiologia brasileira, 2018; 51(4): 211-217.

3. ANDREOLLO NA, et al. A quimiorradioterapia adjuvantes após gastrectomia subtotal e total com linfadenectomia d2 aumentam a sobrevida no câncer gástrico avançado?. ABCD: arquivos brasileiros de cirurgia digestiva, 2019; 32(4): 1-6.

4. BERGERON-BOUCHER MP, et al. Understanding Differences in Cancer Survival between Populations: A New Approach and Application to Breast Cancer Survival Differentials between Danish Regions. International Journal of Environmental Research, 2019; 16(17): 1-16.

5. CHEN K, et al. Mutational landscape of gastric adenocarcinoma in chinese: implications for prognosis na terapy. Proceedings of the National Academy of Sciences, 2015; 112(4): 1107-1112.

6. COSTA WL Jr, et al. Total Gastrectomy for Gastric Cancer: An Analysis Of Postoperative And Long-Term Outcomes Through Time. Gastrointestinal Oncology, 2015; 22(3): 750-757. 
7. DIACONU S, et al. Helicobacter pylori infection: old and new. J Med Life, 2017; 10(2): 112-117.

8. FABIANI L, et al. Influência da presença de metástase no perfil de mortalidade de pacientes oncológicos. Revista Eletrônica Acervo Saúde, 2019; 11(05): 1-8.

9. FIGUEROA-GIRALT M. Factores pronósticos de sobrevida alejada en cáncer gástrico. Introducción del nuevo índice $N_{+} / T$. Revista chilena de cirugía, 2018; 70: 147-59.

10. FIGUEROA-GIRALT, M. Factores pronósticos de sobrevida alejada en cáncer gástrico. Introducción del nuevo índice N+/T. Revista chilena de cirugía, 2018; 70(2): 147-159.

11. HOWLADER N, et al. SEER Cancer Statistics Review, 1975-2014. Bethesda: National Cancer Institute, 2017.

12. HOYOS-VALDELAMAR JC, et al. Caracterización del cáncer gástrico abordado por laparoscopia en un centro del caribe colombiano. Revista Colombiana de Cirurgia, 2020; 35(4): 575-82.

13. INCA. INSTITUTO NACIONAL DE CÂNCER. Coordenação de Prevenção e Vigilância. Estimativa 2018: incidência de câncer no Brasil. 2017. 122p.

14. JEMAL A, et al. Annual Report to the Nation on the Status of Cancer, 1975-2014, Featuring Survival. Journal of the National Cancer Institute, 2017; 109 (9).

15. JURADO-MUÑOZ PA, et al. Resultados tempranos en pacientes con cáncer gástrico sometidos a gastrectomía laparoscópica con intención curativa. Revista Colombiana de Cirurgia, 2021; 36(1): 74-78

16. MONTALVÁN EE, et al. Survival of Gastric Cancer in Western Honduras Pilot study: 2002-2012. Rev méd hondureña, 2017; 85(1): 6-10.

17. MONTIEL-ROA AJ, et al. Sobrevida y calidad de vida en pacientes gastrectomizados por cancer gástrico. Cirugia paraguaya, 2019; 43(1):20-6.

18. NORERO E, et al. Survival and perioperative morbidity of totally laparoscopic versus open gastrectomy for early gastric cancer: analysis from a single Latin American Centre. ABCD Arquivos Brasileiros de Cirurgia Digestiva, 2019; 32(1): $1-5$.

19. PAPENFUSS WA. Morbidity and Mortality Associated with Gastrectomy for Gastric Cancer. Gastrointestinal Oncology, 2014; 21(9): 3008-3014.

20. PARK JY, et al. Prevention strategies for gastric cancer: a global perspective. Clinical Endoscopy, 2014; 47(6): 47889.

21. RAMOS MFKP, et al. Risk factors associated with the development of gastric cancer - case-control study. Revista da Associação Medica Brasileira, 2018; 64(7): 611-619.

22. RAMOS MFKP, et al. Conversion therapy for gastric cancer: expanding the treatment possibilities. ABCD Arquivos Brasileiros de Cirurgia Digestiva. 2019; 32(02): 1-5..

23. SMYTH EC, et al. Gastric cancer: ESMO Clinical Practice Guidelines for diagnosis, treatment and follow-up. Annals of Oncology, 2016; 27(5): 38-49.

24. STEWART BW, WILD CP. World Cancer Report: 2014. Lyon: IARC, 2014.

25. SZOR DJ, et al. Neutrophil-lymphocyte ratio change after curative gastrectomy for gastric cancer: a subgroup analysis. Einstein (São Paulo), 2020; 18: 1-7.

26. TONETO MG, VIOLA L. Current status of the multidisciplinary treatment of gastric adenocarcinoma. ABCD Arquivos Brasileiros de Cirurgia Digestiva, 2018; 31(2): 1-4

27. WANG S, et al. Postoperative complications and prognosis after radical gastrectomy for gastric cancer: a systematic review and meta-analysis of observational studies. World Journal of Surgical Oncology, 2019; 17(1): 1-10. 